\title{
Repetitive Hypoglycemia in Young Rats Impairs Hippocampal Long-Term Potentiation
}

\author{
KELVIN A. YAMADA, NICHOLAS RENSING, YUKITOSHI IZUMI, GABRIEL A. DE ERAUSQUIN, \\ VERED GAZIT, DAVID A. DORSEY, AND DANIEL G. HERRERA \\ Departments of Neurology [K.A.Y., N.R., V.G.] and Psychiatry [Y.I., G.A.D., D.A.D.], and the Center for \\ the Study of Nervous System Injury [K.A.Y., N.R., V.G.] at Washington University School of Medicine; \\ Department of Pediatric Neurology and the Pediatric Epilepsy Center at St. Louis Children's Hospital \\ [K.A.Y.], St. Louis, Missouri 63110, U.S.A.; and Department of Psychiatry, Weill Medical College of \\ Cornell University, New York, New York 10021, U.S.A. [D.G.H.]
}

\begin{abstract}
Mechanisms underlying cognitive dysfunction in young diabetic children are poorly understood, and may include synaptic dysfunction from insulin-induced hypoglycemia. We developed a model of repetitive insulin-induced hypoglycemia in young rats and examined hippocampal long-term potentiation, an electrophysiologic assay of synaptic plasticity, 3-5 d after the last hypoglycemic event. Three hypoglycemic events between postnatal d 21-25 produced modest cortical ( $17 \pm 2.9$ dead neurons per section in parasagittal cortex), but not hippocampal, neuron death quantified by Fluoro-Jade B staining. There was no change in neurogenesis in the hippocampal dentate granule cell region by quantification of bromodeoxyuridine incorporation. Although normal baseline hippocampal synaptic responses were elicited from hippocampal slices from hypoglycemic animals, long-term synaptic potentiation could not be induced in hippocampal slices from rats subjected to hypoglycemia. These results suggest that repetitive hypoglycemia in the developing brain can cause selective impairment of synaptic plasticity in the absence of cell death,
\end{abstract}

\section{ABSTRACT}

and without complete disruption of basal synaptic transmission. We speculate that impaired synaptic plasticity in the hippocampus caused by repetitive hypoglycemia could underlie memory and cognitive deficits observed in young diabetic children, and that cortical neuron death caused by repetitive hypoglycemia in the developing brain may contribute to other neurologic, cognitive, and psychological problems sometimes encountered in diabetic children. (Pediatr Res 55: 372-379, 2004)
Abbreviations
LTP, long-term potentiation
Hex, hexamethonium chloride
HI, hexamethonium chloride plus insulin
EPSP, excitatory postsynaptic potential
BrdU, bromodeoxyuridine
PBS+, PBS with $10 \%$ goat serum and $0.3 \%$ Triton $\mathrm{X}$
DAPI, 4',6-diamidino-2-phenylindole

Diabetic children diagnosed before age $5 \mathrm{y}$ are at risk for lasting cognitive impairment (1), and hypoglycemia may be a causative factor $(2,3)$. Young children on conventional therapy are prone to hypoglycemic events $(4-8)$, which occur even more frequently on intensive therapy $(9,10)$. Severe hypoglycemia was a risk factor for cognitive deficits in one prospective study (11), but not another (12), raising the possibility that less severe, repetitive hypoglycemia (not evaluated in these studies)

Received March 14, 2003; accepted October 15, 2003.

Correspondence: Kelvin A. Yamada, M.D., Department of Neurology, Box 8111, Washington University School of Medicine, 660 South Euclid Avenue, St. Louis, MO 63110, U.S.A.; e-mail: YamadaK@neuro.wustl.edu

Supported by NIH grants DK20579 and NS042774.

DOI: 10.1203/01.PDR.0000110523.07240.C1 causes cognitive impairment. Moreover, continuous glucose monitoring reveals that prolonged nocturnal hypoglycemia occurs frequently in children with type 1 diabetes $(13,14)$. The mechanisms by which diabetes or hypoglycemia produce relatively subtle cognitive deficits without overt brain injury are poorly understood, and these two factors are difficult to study independently in diabetic children. Therefore, we developed a model of hypoglycemia-induced brain injury in developing rats to simulate repetitive hypoglycemia as encountered in diabetic children to study the consequences of repetitive hypoglycemia at a cellular level under more controlled experimental conditions. Repetitive hypoglycemia between postnatal d 21 and 25 impaired hippocampal LTP, without causing hippocampal neuron death. Because LTP is a candidate cellular mechanism 
underlying learning and memory (15), we hypothesize that hypoglycemia could disrupt hippocampal synaptic plasticity during brain development in diabetic children, and contribute to cognitive impairment. Recent studies suggest that children with type 1 diabetes who experience hypoglycemia exhibit impairment of hippocampal-dependent memory $(10,16)$.

\section{METHODS}

Hypoglycemia. Experiments were performed on SpragueDawley rats allowed food and water ad libitum. Figure $1 \mathrm{~A}$ shows the timeline for the 7- to 11-d experimental period. Figure $1 B$ shows the timeline for hypoglycemic events, which animals experienced on three different days with an intervening recovery day. On postnatal d 21, 23, and 25 all rats received 30 $\mathrm{mg} / \mathrm{kg}$ of the ganglion blocker Hex in normal saline i.p. to reduce autonomic counter-regulatory responses (17), enabling more consistent hypoglycemia with less insulin, facilitating reversal of hypoglycemia, and reducing mortality. This dose was chosen from a study in mice demonstrating that $30 \mathrm{mg} / \mathrm{kg}$ Hex almost completely eliminates the rise of immunoreactive glucagon produced by insulin-induced hypoglycemia to the range of $18 \pm 2 \mathrm{mg} / \mathrm{dL}$ (18). Fifteen minutes later, hypoglycemic rats (HI) received $10-15 \mathrm{U} / \mathrm{kg}$ of insulin lispro (Humalog, Eli Lilly, Indianapolis, IN, U.S.A.). Hex controls received saline instead of insulin. Insulin doses were based on preliminary experiments, combined with behavioral observation and EEG. In these preliminary studies, some rats were anesthetized with halothane, and epidural screw electrodes were placed in the skull using aseptic technique and secured with dental

\begin{tabular}{|c|c|c|c|c|c|}
\hline P21 & P22 & P23 & P24 & P25 & P28-32 \\
\hline $\begin{array}{l}\text { Hex insulin } \\
\text { or sham }\end{array}$ & Recovery & $\begin{array}{l}\text { Hex insulin } \\
\text { or sham }\end{array}$ & Recovery & $\begin{array}{l}\text { Hex insulin } \\
\text { or sham }\end{array}$ & $\begin{array}{l}\text { Electrophysiology } \\
\text { Histology }\end{array}$ \\
\hline
\end{tabular}
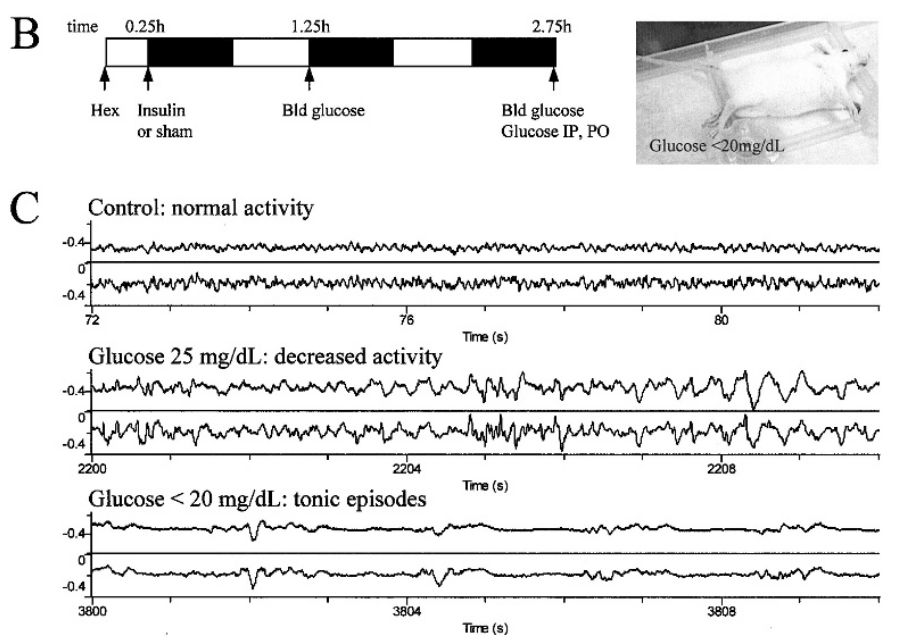

Figure 1. Hypoglycemia in postnatal d $(P)$ 21-25 rats. $A$, timeline for the experimental model. $B$, timeline for drug injection and glucose (Bld glucose) measurements during insulin-induced hypoglycemic events. Photograph shows a rat exhibiting a tonic episode when blood glucose was $<20 \mathrm{mg} / \mathrm{dL}$. $C, 10$-s samples from two-channel EEG recordings (left and right hemisphere) from a rat showing normal 6 - to $8-\mathrm{Hz}$ brain wave activity during euglycemia, delta (1-3 Hz) slowing with hypoglycemia, and periodic complexes but not isoelectricity or seizures during tonic episodes associated with blood glucose below $20 \mathrm{mg} / \mathrm{dL}$. cement, two over the right and two over the left hemisphere (19). Blood glucose was measured with a commercial glucometer (Freestyle, Therasense, Inc., Alameda, CA, U.S.A.; detection range $20-500 \mathrm{mg} / \mathrm{dL}$ ) $1 \mathrm{~h}$ after saline injection in Hex controls, and 1 and $2.5 \mathrm{~h}$ after insulin injection during hypoglycemia. For statistical analysis blood glucose $<20 \mathrm{mg} / \mathrm{dL}$ was assigned a value of $19 \mathrm{mg} / \mathrm{dL}$. Hypoglycemia was terminated after the second blood glucose measurement by oral administration of $50 \%$ dextrose in water and returning the animal to solid food. If animals were unable to eat or drink, 0.1 $\mathrm{mL}$ of $50 \%$ dextrose in water was given i.p., enabling resumption of feeding. Animal care and experimentation conformed to Public Health Service Guide for Care and Use of Laboratory Animals and American Veterinary Medical Association Panel on Euthanasia Guidelines, and were approved by the Washington University School of Medicine Animal Studies Committee.

Histology. To quantify cell death, on postnatal d 28 rats were anesthetized with halothane and perfused intracardiac with ice-cold saline buffer followed by $4 \%$ paraformaldehyde with $0.025 \%$ glutaraldehyde in PBS. Brains were postfixed $\left(4^{\circ} \mathrm{C}\right)$ overnight then cryoprotected in PBS with 30\% sucrose overnight. Forty-micron coronal sections were prepared with a freezing sliding microtome and stained with Fluoro-Jade B (HistoChem, Jefferson, AR, U.S.A.) and DAPI as described by Schmued and Hopkins (20). Fluoro-Jade B-positive dead neurons were visually identified and manually counted in predetermined regions using epifluorescence microscopy (xenon light source, Chroma filter sets, UV-2 for DAPI and FITC for Fluoro-Jade B, $16 \times 0.45 \mathrm{NA}$ objective) in sections approximately $3.0 \mathrm{~mm}$ posterior to bregma (Fig. $2 A$ ). Fluoro-Jade B-positive neurons were counted in a $1.5 \times 1.0 \mathrm{~mm}$ rectangular region encompassing the midline and parasagittal cortex, or within the temporal lobe from the entorhinal fissure to the medial extent of the temporal lobe cortex (rectangular regions in Fig. 2A). The entire hippocampus, thalamus, and hypothalamus within each section were surveyed for Fluoro-Jade B-positive neurons.

To quantify cell proliferation in the dentate granule cell layer, animals were injected with $100 \mathrm{mg} / \mathrm{kg} \mathrm{BrdU}$ in water i.p. $45 \mathrm{~min}$ before Hex injection on postnatal d 21, 23, and 25, or at $1300 \mathrm{~h}$ on postnatal d 22, 24, and 26-27. On postnatal d 28 rats were perfusion fixed and brains postfixed as described above, but the brains were not cryoprotected. Serial $50-\mu \mathrm{m}$ coronal sections (total 55-60 per brain) were prepared with a vibratome and immunolabeled for BrdU. For BrdU labeling, sections were incubated in $50 \%$ formamide $-2 \times \mathrm{SSC}$ (sodium chloride citrate) for $1 \mathrm{~h}$ at $55^{\circ} \mathrm{C}$. After a rapid wash with $2 \times \mathrm{SSC}$, they were incubated in $2 \mathrm{~N} \mathrm{HCl}$ for $30 \mathrm{~min}$ at room temperature followed by washes in $0.1 \mathrm{M}$ boric acid for $2 \mathrm{~min}$ and then washed three times in PBS. Sections were blocked in PBS + for $1 \mathrm{~h}$ at room temperature. Endogenous peroxide activity was blocked by incubating for $30 \mathrm{~min}$ in a solution of $3 \%$ peroxide in methanol; the sections were then incubated overnight in rabbit anti-BrdU (Megabase Research Products, Lincoln, NB, U.S.A.; diluted 1:500 in PBS+). Sections were washed three times in PBS, incubated overnight in secondary antibody (goat anti-rabbit $\operatorname{IgG}(\mathrm{H}+\mathrm{L})$-horseradish peroxidase 
A
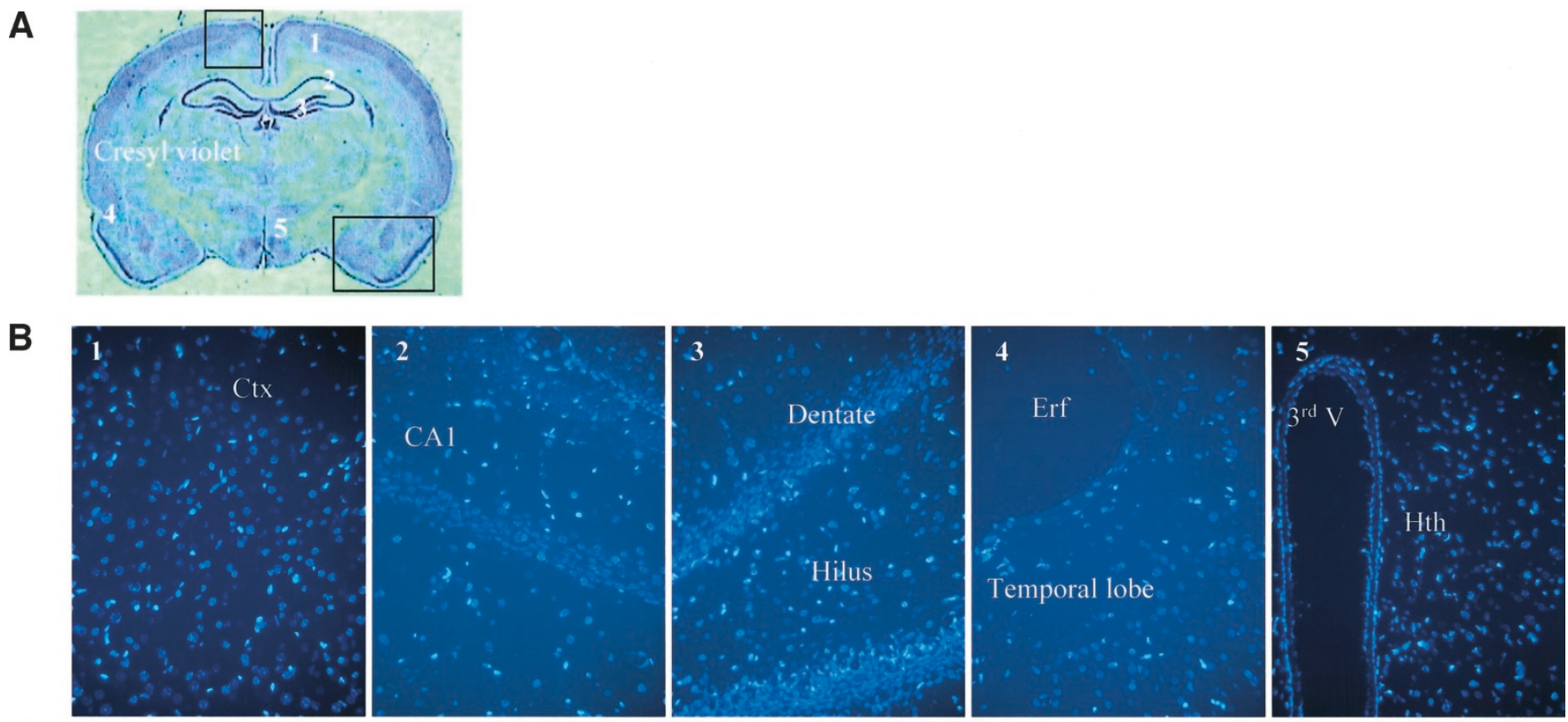

C
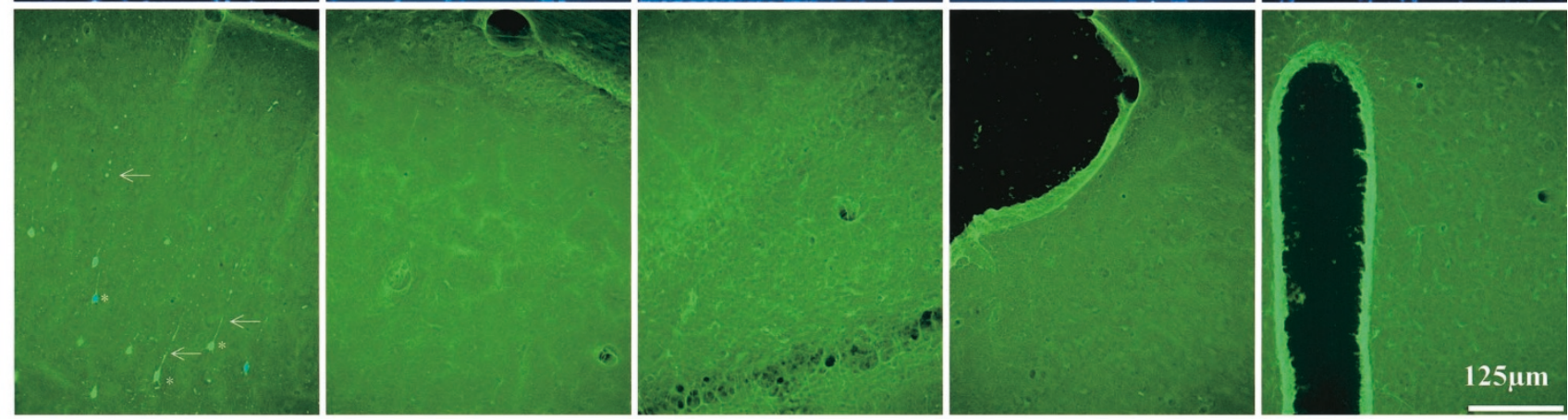

Figure 2. Double staining with Fluoro-Jade B and DAPI demonstrate hypoglycemia-induced cortical neuron death. $A$, numbers in this cresyl violet-stained coronal section indicate locations of representative regions shown in $B$ and $C$, where quantification of neuronal death was performed. $B$, DAPI-positive live neurons are seen in all sections. $C t x$, cortex; Erf, entorhinal fissure; $3 r d V$, third ventricle; $H t h$, hypothalamus. $C$, same sections in $B$ viewed with a FITC filter. Fluoro-Jade B-positive dead neurons (asterisks adjacent to three of the 10 neurons in this field) and degenerating neurites (punctate and linear fluorescence, arrows) are confined to cortical regions, and are absent in the hippocampus, temporal lobe, and hypothalamus. All images are identically oriented, top is dorsal; calibration bar applies to all fluorescent images.

conjugate), diluted 1:1000 in PBS + , and washed three times in PBS. Fast 3,3'-diaminobenzidine (Sigma D0426; Sigma Chemical Co, St. Louise, MO, U.S.A.) cobalt-enhanced was applied for $30 \mathrm{~s}$ at room temperature.

BrdU-positive cells in the hippocampal dentate gyrus were counted using unbiased stereologic methods. For each animal BrdU cells were counted in every sixth section (typically nine sections per animal), using a $20 \mu \mathrm{m} \times 20 \mu \mathrm{m}$ grid with a $\times 100$ microscope objective. The granule cell layer of the dentate gyrus was measured using a semiautomatic stereology system. The volume of the entire dentate granule cell layer was calculated according to the Cavalieri estimation (21).

Electrophysiology. Rats were killed on postnatal d 28-32 for transverse hippocampal slice preparation and recording (22). After incubation slices were transferred to a submersion recording chamber continuously perfused with artificial cerebrospinal fluid containing (in millimolar): $124 \mathrm{NaCl}, 5 \mathrm{KCl}, 2$ $\mathrm{MgSO}_{4}, 2 \mathrm{CaCl}_{2}, 1.25 \mathrm{NaH}_{2} \mathrm{PO}_{4}, 22 \mathrm{NaHCO}_{3}$, and 10 glucose, bubbled with $95 \% \mathrm{O}_{2}-5 \% \mathrm{CO}_{2}$ at $30^{\circ} \mathrm{C}$. Extracellular recordings were obtained from the pyramidal layer and stratum radiatum of CA1. Bipolar constant-current pulses $(0.1-0.2 \mathrm{~ms})$ sufficient to evoke a half-maximal population spike were applied to the Schaffer collateral-commissural fibers to elicit EPSPs. EPSP slopes were normalized to the EPSP slope averaged from a baseline recording period, and presented as a percentage of the baseline EPSP slope. LTP was induced using a $1-\mathrm{s}, 100-\mathrm{Hz}$ tetanic stimulus. Successful induction and maintenance of LTP was defined as $>20 \%$ increase of EPSP slope over baseline, $60 \mathrm{~min}$ after tetanus.

Values are mean \pm SEM unless otherwise indicated. Statistical significance was assessed with the Mann-Whitney $U$ test or $t$ test using $p<0.05$ to reflect statistically significant differences between groups. Chemicals were from Sigma Chemical Co. (St. Louis, MO, U.S.A.) except as indicated.

\section{RESULTS}

A total of 83 rats were used (56 hypoglycemic and 27 controls); one rat died after its second hypoglycemic event, a $1.8 \%$ mortality for the hypoglycemia group. Thirty-six (26 HI 
and 10 Hex) rats were killed on postnatal d 28 for Fluoro-Jade B examination. Ten HI rats were killed on postnatal d 28 (two rats), postnatal d 35 (four rats), and postnatal d 42 (four rats) for Fluoro-Jade B examination to determine whether cell death occurred beyond $72 \mathrm{~h}$ after the last hypoglycemic event. Twenty (10 HI, $10 \mathrm{Hex}$ ) rats were killed on postnatal d 28 for BrdU labeling, but two HI brains were damaged during sectioning and could not be processed for analysis, leaving eight $\mathrm{HI}$ and $10 \mathrm{Hex}$ brains for BrdU studies. Sixteen (nine HI, four Hex, three sham) rats were killed on postnatal d 28-32 for hippocampal LTP studies. Four of the nine hypoglycemic animals used for LTP studies received $10 \mathrm{U} / \mathrm{kg}$ of insulin; all other hypoglycemic animals received $15 \mathrm{U} / \mathrm{kg}$ insulin. The target blood glucose was $\leq 35 \mathrm{mg} / \mathrm{dL}$; the mean blood glucose for 330 measurements from $55 \mathrm{HI}$ rats was $43 \pm 32$ (1 SD) $\mathrm{mg} / \mathrm{dL}$, and $68 \%$ of blood glucose measurements were $\leq 35$ $\mathrm{mg} / \mathrm{dL}$. In pilot studies these doses of insulin alone produced inconsistent achievement of target hypoglycemia, and higher doses of insulin alone resulted in difficult to reverse hypoglycemia and increased mortality. Therefore, we used autonomic ganglion blockade by Hex to inhibit the counter-regulatory responses to hypoglycemia, and we were able to achieve more consistent hypoglycemia and observed dramatically improved survival. We did not observe hypoglycemia in rats treated with Hex alone. Rats were usually asymptomatic until blood glucose was $<40 \mathrm{mg} / \mathrm{dL}$, when they became less active. Below 20 $\mathrm{mg} / \mathrm{dL}$ animals typically lost posture and exhibited episodic tonic extension of the limbs lasting a few seconds at a time (Fig. $1 B$, photo). To determine whether these episodes were seizures, which might contribute to neuronal injury, in some rats EEG recordings were obtained before and during moderate and severe hypoglycemia. During severe hypoglycemia the EEG showed slower frequency brain wave activity than during euglycemia, but during tonic episodes there was no seizure activity (Fig. 1C).

Fluoro-Jade B-positive dead neurons were counted in the five brain regions labeled in Figure 2A. DAPI staining shows nuclei of live cells (Fig. $2 B$ ) in the same sections also stained with Fluoro-Jade B (Fig. 2C). Neuronal death was confined to cortical regions with rare exceptions, predominantly in the parasagittal cortex approximately $1 \mathrm{~mm}$ lateral to midline (Fig. $2 C$, left $)$ and to a lesser extent in the temporal lobes ( $p<$ 0.001, Mann-Whitney $U$ test; Fig. 4A, 5A-C), whereas neuronal death was extremely rare in the hippocampus and thalamus or hypothalamus (Figs. $2 C$ and $4 A$ ). Dead neurons were observed in the temporal lobes of Hex controls $(4.3 \pm 1.2$ Fluoro-Jade B-positive neurons, $n=10$ not shown), and in controls that did not receive Hex (5.3 \pm 0.6 Fluoro-Jade B-positive neurons, $n=3$ not shown), possibly reflecting tissue damage from brain removal during our efforts to protect the dorsal cortex and hippocampus. The average number of Fluoro-Jade B-positive dead neurons for each brain region from $26 \mathrm{HI}$ animals are presented in Figure $4 A$. In regions of neuronal death, linear and punctate Fluoro-Jade B staining also demonstrated degenerating neurites (Fig. $2 C$ ), which are shown more clearly in a higher power image (Fig. 3, $A$ and $C$ ). DAPI staining from the same high-power sections shows nuclei from live neurons (Fig. 3, $B$ and $D$ ).

All $27 \mathrm{HI}$ rats (including the one death) experienced at least one hypoglycemic event in which blood glucose was $<50$ $\mathrm{mg} / \mathrm{dL}$ at both 1 and $2.5 \mathrm{~h}$. The average blood glucose for all measurements was $51 \pm 36 \mathrm{mg} / \mathrm{dL}(\mathrm{SD}, n=160)$; Figure $4 B$
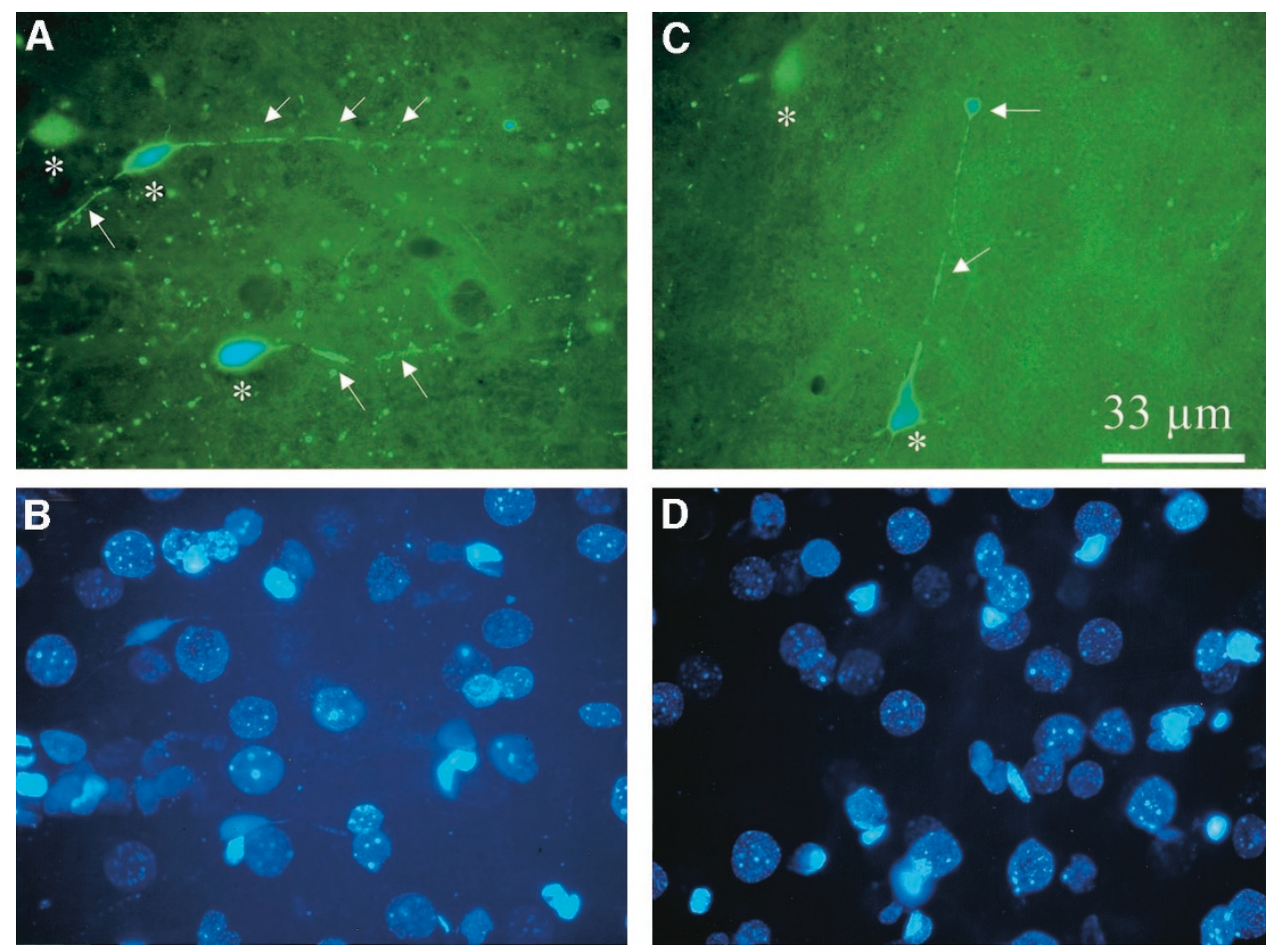

Figure 3. Fluoro-Jade $\mathrm{B}$ demonstrates neuronal death and degenerating neurites after hypoglycemia. $A$ and $C$, Fluoro-Jade B-positive dead neurons (asterisks) and punctate Fluoro-Jade B fluorescence indicative of degenerating neurites in two parasagittal cortical regions. Linear fluorescence (arrows) are degenerating dendrites. $B$ and $D$, DAPI staining of the same section demonstrates nuclei from live neurons. $\times 60,1.3 \mathrm{NA}$; scale bar applies to all images. 
A

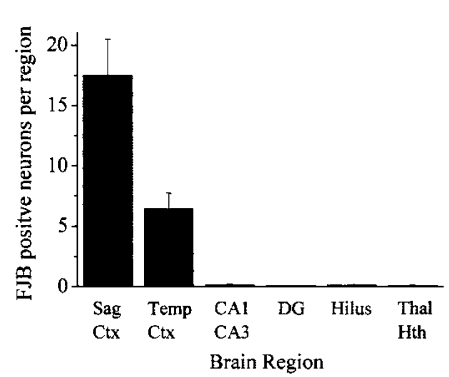

B

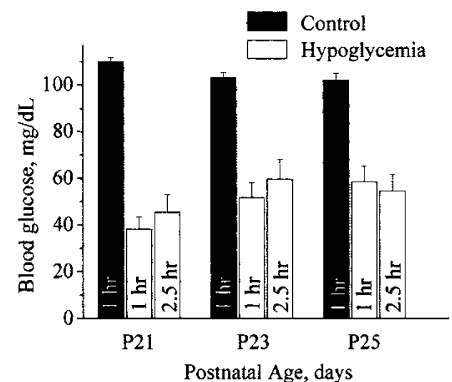

$\mathrm{C}$

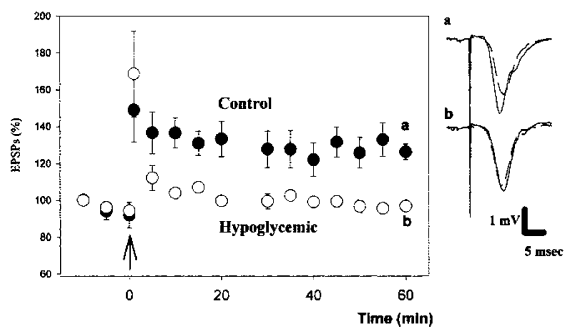

$\mathrm{D}$

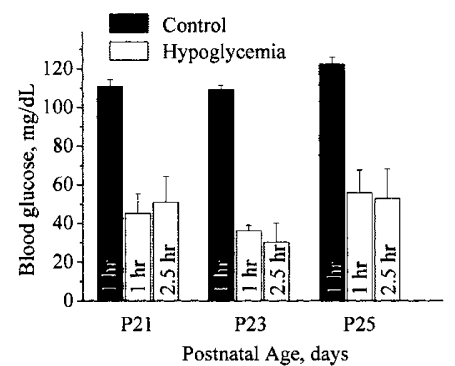

Figure 4. Insulin-induced hypoglycemia produces neuronal death and lasting impairment of hippocampal long-term potentiation. $A$, averages of Fluoro-Jade B $(F J B)$-positive dead neurons in different brain regions (see Fig. $2 A$ ) from 26 hypoglycemic animals examined histologically. $B$, blood glucose levels in rats from $A$ show hypoglycemia in insulin-treated rats (different days, NS) and euglycemia in saline-treated rats. $C$, electrically evoked EPSPs recorded from hippocampal slices obtained from control and hypoglycemic animals. The arrow indicates time of LTP induction by tetanus. Control slices, closed circles (a) show successful LTP induction and maintenance. Slices from hypoglycemic animals, open circles (b) show no LTP induction; none of the 17 slices had potentiation over baseline. Representative EPSP tracings $60 \mathrm{~min}$ after tetanus (solid lines) superimposed on the baseline EPSP (broken line) show LTP for the control slice (a) but not the slice from the hypoglycemic rat $(b)$. $D$, blood glucose measurements from control and hypoglycemic animals used for LTP studies are comparable to those from histologic studies shown in $B$. Sag Ctx, sagittal cortex; temp ctx, temporal lobe cortex; DG, dentate granule; Thal, thalamus; Hth, hypothalamus.

shows the averages of the blood glucose measurements obtained during the three hypoglycemic events (NS). The large SD reflects that hypoglycemia sometimes did not occur $(33 \%$ of the 160 measurements were above $50 \mathrm{mg} / \mathrm{dL}$ ). In $10 \mathrm{Hex}$ control rats the average blood glucose $1 \mathrm{~h}$ after injections was $102 \pm 9.5 \mathrm{mg} / \mathrm{dL}(\mathrm{SD}, n=30)$, and Fluoro-Jade B-positive dead neurons were not observed in the parasagittal cortex, hippocampus, and thalamus or hypothalamus of any of the sections (Fig. 2C).

EPSP slopes, population spike amplitude, and population spike input-output relationship to stimulus intensity were comparable in hippocampal slices from hypoglycemic and control rats, indicating that basal synaptic transmission was comparable between groups (not shown). However, LTP could not be induced in any of the 17 slices from nine hypoglycemic animals (EPSP slope $93 \pm 3 \%$ of baseline, compared with an average of $124 \pm 9$ in nine slices from seven Hex and sham controls), indicating impairment of this form of synaptic plasticity ( $p=0.003, t$ test; Fig. $4 C)$. There was no difference in LTP between the Hex and sham controls. In animals used for LTP studies hypoglycemia was comparable to that achieved in histology studies; $76 \%$ of blood glucose measurements were $<50 \mathrm{mg} / \mathrm{dL}$ for HI (average $46 \pm 34 \mathrm{mg} / \mathrm{dL}$ combining 1 and $2.5 \mathrm{~h}$ after insulin, SD, $n=54)$, compared with $114 \pm 10$ $\mathrm{mg} / \mathrm{dL}$ (SD, $n=21$ ) for Hex controls (Fig. $4 D$ ).

Because LTP recordings were performed on postnatal $d$ 28-32, delayed cell death after postnatal d 28 may have caused impaired LTP and would not have been detected by our histologic analysis on postnatal d 28. Therefore, we subjected another litter of 10 rats to three hypoglycemic events as described previously, and allowed animals to survive to postnatal d $28(n=2)$, postnatal d $35(n=4)$, and postnatal d $42(n$ $=4$ ) before sacrifice for Fluoro-Jade B and DAPI staining and quantification of cell death. All of the animals had at least two of their three hypoglycemic events in the target range of hypoglycemia. Fewer dead neurons were observed in sections prepared at postnatal d 35 and postnatal d 42 compared with postnatal d 28 (Fig. 5, $A-C$ ), and the fluorescence intensity of positive cells also decreased (not shown), suggesting that ongoing or delayed cell death was unlikely. It also suggests that dead cells that would have been observed on postnatal d 28 may have been eliminated by postnatal $\mathrm{d} 35$ and postnatal d 42 , as observed using Fluoro-Jade B staining after kainic acidinduced hippocampal neuron death (23).

Postnatal neurogenesis occurs in the dentate gyrus of the hippocampus $(24,25)$, and it is possible this process is involved in hippocampal development, plasticity, and response to injury. Consequently, we tested the hypothesis that repetitive hypoglycemia would reduce hippocampal neurogenesis as is observed in developing rats subjected to repetitive seizures (26). We also hypothesized that an alteration in hippocampal development might account for impaired LTP. Postnatal d 21 rats were subjected to hypoglycemia three times by HI treatment $(n=8)$ or given only Hex and sham-treated $(n=10)$ as described above. We observed comparable hypoglycemia (Fig. 6D) to experiments for Fluoro-Jade B quantification of neuronal death (Fig. 4B), LTP experiments (Fig. 4D), and assessment of 
A

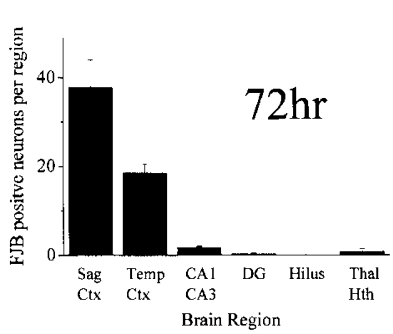

D

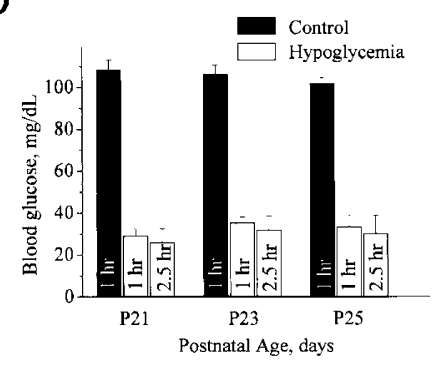

B

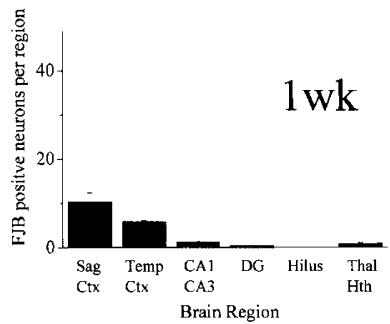

$\mathrm{C}$

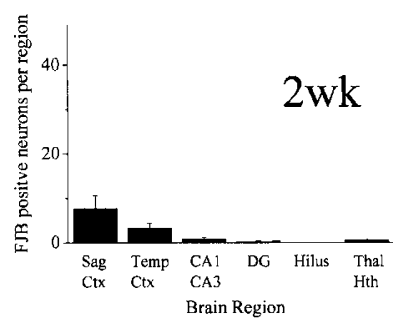

Figure 5. Delayed cell death does not occur after repetitive insulin-induced hypoglycemia. Ten animals from the same litter were subjected to three hypoglycemic events as schematized in Figure $1, A$ and $B$, and allowed to survive $3 \mathrm{~d}$ [postnatal d $(P) 28, n=2$ ], 1 wk $(\mathrm{P} 35, n=4)$, or 2 wk $(\mathrm{P} 42, n=4)$ after the last hypoglycemic event. Neuronal death was quantified by Fluoro-Jade B or DAPI staining as shown in Figures 2-4. Compared with 3 d after hypoglycemia $(A)$, less neuronal death is observed $1(B)$ and $2(C)$ wk after hypoglycemia, and the fluorescence intensity of positively stained cells diminished (not shown). Decreasing numbers of Fluoro-Jade B-stained neurons argues against ongoing or delayed neuronal death after hypoglycemia in this model. $D$, note that slightly lower average blood glucose concentrations were achieved in this experiment (compare with Fig. 4B), perhaps accounting for more neuronal death at $3 \mathrm{~d}(A)$ compared with Figure $4 A$. Sag Ctx, sagittal cortex; temp ctx, temporal lobe cortex; DG, dentate granule; Thal, thalamus; Hth, hypothalamus.

A

B
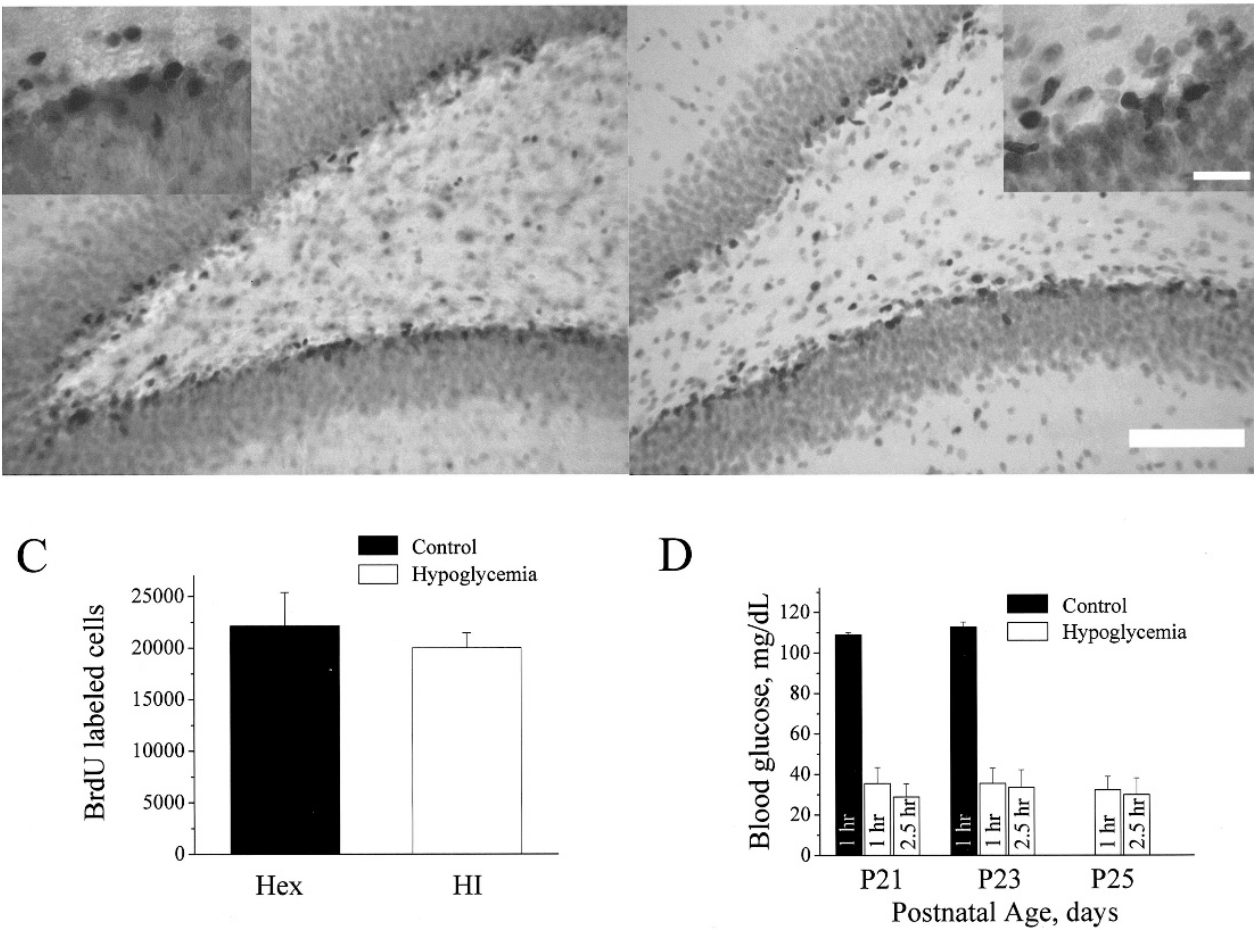

Figure 6. Repetitive hypoglycemic events do not affect neurogenesis in the hippocampal dentate granule cell layer. Light microscopic images of the hippocampal dentate granule cell layer and hilus demonstrates darkly stained cells immunolabeled for BrdU $(A, B) ; \times 16$, calibration $100 \mu \mathrm{m}$. Insets are higher magnification of part of the same section $(\times 100$, calibration $20 \mu \mathrm{m})$. Representative sections show BrdU-positive cells in Hex-treated control sections $(A)$ compared with sections from a hypoglycemic animal treated with $\mathrm{HI}(B)$. $C$, cumulative quantitative data from 18 rats (10 Hex and eight $\mathrm{HI})$ show no significant differences in numbers of BrdU-labeled cells per entire dentate gyrus volume in control compared with hypoglycemic rats $(p=0.55)$. $D$, average blood glucose levels verify successful induction of hypoglycemia in HI-treated animals. $P$, postnatal $\mathrm{d}$. 
delayed cell death (Fig. 5D). As before (Fig. 1A), animals were killed on postnatal $\mathrm{d} 28$ for processing. There were no significant differences in BrdU-immunolabeled cells (Fig. 6, $A$ versus $B$ ) in sections from HI- compared with Hex-treated animals. Figure $6 D$ shows cumulative data from the 18 animals analyzed, indicating that comparable numbers of BrdU-labeled cells were counted per entire dentate gyrus volume in Hex- and HI-treated animals $(p=0.55)$.

\section{DISCUSSION}

We subjected young rats to repetitive hypoglycemic events to simulate hypoglycemia encountered in diabetic children. Our ultimate goal is to study and understand mechanisms of brain dysfunction from hypoglycemia during CNS development in the setting of diabetes. Important features of this model are the extent and duration of repetitive hypoglycemic events, which are comparable to what has been observed in diabetic children using continuous glucose monitoring $(13,14)$. We used Hex to block autonomic counter-regulatory responses for two reasons. The first was to improve consistency of hypoglycemia and reduce mortality (mortality from hypoglycemia is low in diabetic children). Approximately two thirds of the three hypoglycemic events produce blood glucose $<35 \mathrm{mg} / \mathrm{dL}$, and only one of 56 hypoglycemic animals died. Second, there is evidence that counter-regulatory responses to hypoglycemia may be impaired in diabetic children, especially those who experience hypoglycemia (27). Autonomic ganglion blockade simulates impairment of counter-regulatory response to hypoglycemia.

We tested the hypothesis that hypoglycemia in the developing rat would cause hippocampal injury, resulting in abnormal synaptic plasticity. Unexpectedly, we did not observe neuronal death in the hippocampus (or hypothalamus or thalamus), and we observed only modest but variable neuronal loss predominantly in the parasagittal and temporal cortex. A notable feature of this model is that although synaptic transmission is normal in hippocampal slices from hypoglycemic rats compared with control rats, synaptic plasticity assessed by LTP induction at CA1 synapses is completely disrupted in hypoglycemic rats 3-6 d after the last hypoglycemic event. We examined LTP at hippocampal synapses because it is regarded a candidate cellular mechanism underlying spatial memory (15), and diabetic children exhibit spatial memory deficits (16). An important caveat is that LTP impairment is not equivalent to impaired learning and memory; however, there are many examples in which both impaired LTP and impaired spatial learning are observed, including in diabetic rats tested by the Morris water maze (28). It should be noted that insulin administration in this study recovered LTP in diabetic rats (28), suggesting that insulin administration in our experiments is unlikely to have been the cause of the LTP impairment we observed. The absence of destructive injury to the hippocampus with prominent and lasting functional abnormalities is important because it suggests that impaired synaptic structure and function probably account for LTP impairment. It remains to be determined by future studies whether hypoglycemic animals exhibit impaired performance on hippocampal- dependent spatial learning and memory such as the Morris water maze. Our observations also raise the possibility that recovery or compensation for the abnormalities is possible if the synaptic structural or functional derangements can be corrected.

The absence of hippocampal neuronal injury is strikingly different from the extensive cortical and hippocampal neuron injury most prominently observed in the dentate granule cell layer of mature rats subjected to hypoglycemia (29). One possible explanation for the difference is that the adult rats received single hypoglycemic events sufficient to produce isoelectricity on EEG (29). Even when our animals exhibited episodic tonic posturing associated with blood glucose $<20$ $\mathrm{mg} / \mathrm{dL}$, electrographic seizures or isoelectric EEG activity were not observed. Our results suggest that in postnatal d 21 rats, cortical neurons are more susceptible to hypoglycemiainduced death than hippocampal neurons or hypothalamic or thalamic neurons. The severity of the cell death is relatively mild, but the most consistently and severely damaged region is the parasagittal cortex about $1 \mathrm{~mm}$ lateral to midline. No neuronal death was observed in the parasagittal cortex of Hex or sham controls. The significance of cortical neuron death in our hypoglycemia model is unclear, but we speculate that if this subtle degree of cortical neuron injury occurs in diabetic children who experience repetitive hypoglycemia, then it might be the pathologic substrate for cognitive, affective, and attention deficits in some diabetic children.

In adult rodents, hippocampal dentate granule cell neurogenesis assessed by BrdU incorporation into mitotically active cells and their progeny is increased by seizures (30), ischemia (31), and trauma (32). Interestingly, in contrast, seizures in developing rats produce a decrease in neurogenesis in the dentate gyrus (26). It is not known how seizures in developing rats cause reductions in neurogenesis, or whether there is a functional correlate. In our hypoglycemia model, the absence of hypoglycemia-induced cell death in the hippocampus by Fluoro-Jade B staining, and the absence of alterations of BrdU-labeled cells in the hippocampal dentate granule cell layer, also suggests that repetitive hypoglycemia did not affect dentate granule cell neurogenesis. This further supports our conclusion that synaptic functional alterations instead of neuronal death account for the impairment in hippocampal synaptic plasticity.

\section{CONCLUSIONS}

These studies were motivated by observations that diabetic children younger than 5-6 y of age are at risk for lasting cognitive impairment. Some studies have identified subtle and relatively specific memory impairment, perhaps reflecting temporal or hippocampal dysfunction $(16,33)$. From our results, we hypothesize that despite minimal hypoglycemia-induced hippocampal neuron death, substantial impairment of synaptic plasticity may account for susceptibility of the youngest diabetic children to learning and memory impairment. Our results may provide insight into potential relationships between hypoglycemia-induced hippocampal dysfunction and cognitive impairment, and may allow studying the effects of diabetes and 
hypoglycemia independently and together in a clinically relevant animal model.

Acknowledgments. The authors thank Dr. Tamara Hershey, Dr. Philip Cryer, Dr. Steven Rothman, and Dr. Mark Goldberg for helpful discussions. We are grateful to Dr. Larry Schmued for advice on using Fluoro-JadeB.

\section{REFERENCES}

1. Northam EA, Anderson PJ, Werther GA, Warne GL, Adler RG, Andrewes D 1998 Neuropsychological complications of IDDM in children 2 years after disease onset. Diabetes Care 21:379-384

2. Rovet JF, Ehrlich RM 1999 The effect of hypoglycemic seizures on cognitive function in children with diabetes: a 7-year prospective study. J Pediatr 134:503-506

3. Ryan CM, Becker DJ 1999 Hypoglycemia in children with type 1 diabetes mellitus Risk factors, cognitive function, and management. Endocrinol Metab Clin North Am 28:883-900

4. Davis EA, Keating B, Byrne GC, Russell M, Jones TW 1998 Impact of improved glycaemic control on rates of hypoglycaemia in insulin dependent diabetes mellitus. Arch Dis Child 78:111-115

5. Hagen JW, Barclay CR, Anderson BJ, Feeman DJ, Segal SS, Bacon G, Goldstein GW 1990 Intellective functioning and strategy use in children with insulin-dependent diabetes mellitus. Child Dev 61:1714-1727

6. Rovet JF, Ehrlich RM, Hoppe M 1987 Intellectual deficits associated with early onset of insulin-dependent diabetes mellitus in children. Diabetes Care 10:510-515

7. Daneman D, Frank M, Perlman K, Tamm J, Ehrlich R 1989 Severe hypoglycemia in children with insulin-dependent diabetes mellitus: frequency and predisposing factors. J Pediatr 115:681-685

8. Ryan C, Vega A, Drash A 1985 Cognitive deficits in adolescents who developed diabetes early in life. Pediatrics 75:921-927

9. [No authors listed] 1997 Hypoglycemia in the Diabetes Control and Complications Trial. The Diabetes Control and Complications Trial Research Group. Diabetes 46:271-286

10. Hershey T, Bhargava N, Sadler M, White NH, Craft S 1999 Conventional versus intensive diabetes therapy in children with type 1 diabetes: effects on memory and motor speed. Diabetes Care 22:1318-1324

11. Northam EA, Anderson PJ, Jacobs R, Hughes M, Warne GL, Werther GA 2001 Neuropsychological profiles of children with type 1 diabetes 6 years after disease onset. Diabetes Care 24:1541-1546

12. Schoenle EJ, Schoenle D, Molinari L, Largo RH 2002 Impaired intellectual development in children with type I diabetes: association with $\mathrm{HbA}(1 \mathrm{c})$, age at diagnosis and sex. Diabetologia 45:108-114

13. Boland E, Monsod T, Delucia M, Brandt CA, Fernando S, Tamborlane WV 2001 Limitations of conventional methods of self-monitoring of blood glucose: lessons learned from 3 days of continuous glucose sensing in pediatric patients with type 1 diabetes. Diabetes Care 24:1858-1862
14. Kaufman FR, Austin J, Neinstein A, Jeng L, Halvorson M, Devoe DJ, Pitukcheewanont P 2002 Nocturnal hypoglycemia detected with the Continuous Glucose Monitoring System in pediatric patients with type 1 diabetes. J Pediatr 141:625-630

15. Malenka RC, Nicoll RA 1999 Long-term potentiation — a decade of progress? Science 285:1870-1874

16. Hershey T, Lillie R, Sadler M, White NH 2003 Severe hypoglycemia and long-term spatial memory in children with type 1 diabetes mellitus: a retrospective study. J Int Neuropsychol Soc 9:740-750

17. Havel PJ, Veith RC, Dunning BE, Taborsky Jr GJ 1991 Role for autonomic nervous system to increase pancreatic glucagon secretion during marked insulin-induced hypoglycemia in dogs. Diabetes 40:1107-1114

18. Havel PJ, Akpan JO, Curry DL, Stern JS, Gingerich RL, Ahren B 1993 Autonomic control of pancreatic polypeptide and glucagon secretion during neuroglucopenia and hypoglycemia in mice. Am J Physiol 265:R246-R254

19. Wong M, Wozniak DF, Yamada KA 2003 An animal model of generalized nonconvulsive status epilepticus: immediate characteristics and long-term effects. Exp Neurol 183:87-99

20. Schmued LC, Hopkins KJ 2000 Fluoro-jade B: a high affinity fluorescent marker for the localization of neuronal degeneration Brain Res 874:123-130

21. Howard CV, Reed MG 1998 Unbiased Stereology: Three-Dimensional Measurement in Microscopy. Springer Verlag, New York, pp 39-54

22. Izumi Y, Yamada KA, Matsukawa M, Zorumski CF 2003 Effects of insulin on long-term potentiation in hippocampal slices from diabetic rats. Diabetologia 46:1007-1012

23. Hopkins KJ, Wang G, Schmued LC 2000 Temporal progression of kainic acid induced neuronal and myelin degeneration in the rat forebrain. Brain Res 864:69-80

24. Altman J, Das GD 1965 Autoradiographic and histological evidence of postnata hippocampal neurogenesis in rats. J Comp Neurol 124:319-335

25. Gage FH 2000 Mammalian neural stem cells. Science 287:1433-1438

26. McCabe BK, Silveira DC, Cilio MR, Cha BH, Liu X, Sogawa Y, Holmes GL 2001 Reduced neurogenesis after neonatal seizures. J Neurosci 21:2094-2103

27. Barkai L, Vamosi I, Lukacs K 1998 Prospective assessment of severe hypoglycaemia in diabetic children and adolescents with impaired and normal awareness of hypoglycaemia. Diabetologia 41:898-903

28. Biessels GJ, Kamal A, Urban IJ, Spruijt BM, Erkelens DW, Gispen WH 1998 Water maze learning and hippocampal synaptic plasticity in streptozotocin-diabetic rats: effects of insulin treatment. Brain Res 800:125-135

29. Auer RN, Olsson Y, Siesjo BK 1984 Hypoglycemic brain injury in the rat. Correlation of density of brain damage with the EEG isoelectric time: a quantitative study. Diabetes 33:1090-1098

30. Parent JM, Yu TW, Leibowitz RT, Geschwind DH, Sloviter RS, Lowenstein DH 1997 Dentate granule cell neurogenesis is increased by seizures and contributes to aberrant network reorganization in the adult rat hippocampus. J Neurosci 17:37273738

31. Liu J, Solway K, Messing RO, Sharp FR 1998 Increased neurogenesis in the dentate gyrus after transient global ischemia in gerbils. J Neurosci 18:7768-7778

32. Dash PK, Mach SA, Moore AN 2001 Enhanced neurogenesis in the rodent hippocampus following traumatic brain injury. J Neurosci Res 63:313-319

33. Hershey T, Craft S, Bhargava N, White NH 1997 Memory and insulin dependent diabetes mellitus (IDDM): effects of childhood onset and severe hypoglycemia. J In Neuropsychol Soc 3:509-520 